EXTENDED REPORT

\title{
Detecting structural changes in early experimental osteoarthritis of tibial cartilage by microscopic magnetic resonance imaging and polarised light microscopy
}

\author{
H A Alhadlaq, Y Xia, J B Moody, J R Matyas
}

Ann Rheum Dis 2004;63:709-717. doi: 10.1136/ard.2003.011783

\begin{abstract}
See end of article for authors' affiliations

Correspondence to: Associate Professor Y Xia, Department of Physics and Center for Biomedical Research, Oakland University, Rochester, MI 48309, USA; xia@oakland.edu
\end{abstract}

Accepted 4 August 2003

\begin{abstract}
Objectives: To detect changes in the collagen fibril network in articular cartilage in a canine experimental model of early osteoarthritis (OA) using microscopic magnetic resonance imaging ( $\mu$ MRI) and polarised light microscopy (PLM).

Methods: Eighteen specimens from three pairs of the medial tibia of an anterior cruciate ligament transection canine model were subjected to $\mu \mathrm{MRI}$ and PLM study 12 weeks after surgery. For each specimen, the following experiments were carried out: (a) two dimensional $\mu M R I$ images of $T_{2}$ relaxation at four orientations; (b) the tangent Young's modulus; and (c) two dimensional PLM images of optical retardance and fibril angle. Disease induced changes in tissue were examined across the depth of the cartilage at a $\mu \mathrm{MRI}$ resolution of 13.7-23.1 $\mu \mathrm{m}$.

Results: Several distinct changes from $T_{2}$ weighted images of cartilage in $O A$ tibia were seen. For the specimens that were covered at least in part by the meniscus, the significant changes in $\mu M R I$ included a clear shift in the depth of maximum $\mathrm{T}_{2}(21-36 \%)$, a decrease in the superficial zone thickness $(37-38 \%)$, and an increase in cartilage total thickness (15-27\%). These $\mu M R I$ changes varied topographically in the tibia surface because they were not significant in completely exposed locations in medial tibia. The $\mu M R I$ results were confirmed by the PLM measurements and correlated well with the mechanical measurements. Conclusion: Both $\mu M R I$ and PLM can detect quantitatively changes in collagen fibre architecture in early $\mathrm{OA}$ and resolve topographical variations in cartilage microstructure of canine tibia.
\end{abstract}

$\mathrm{O}$ steoarthritis (OA) is a slowly progressive disease of articular cartilage and is considered to be one of most common types of arthritis. ' Several possible events, such as abnormal joint loading, can compromise the integrity of healthy cartilage and result in structural and biochemical changes in the tissue, including alteration in the microstructure and composition of collagen and proteoglycan networks. These changes can eventually produce osteoarthritic-like symptoms including change in cartilage thickness, increase in hydration, and change in biomechanical properties. $^{2}$ At advanced stages of OA, severe fibrillation that ultimately leads to a complete loss of the articular cartilage down to the subchondral bone can be seen in cartilage. The critical role of the collagen matrix in cartilage is to preserve tissue integrity and to maintain the water/proteoglycan environment. Any alteration of the collagen microstructure will inevitably disrupt the molecular environment of collagen fibrils, proteoglycans, and water, consequently modifying the mechanical properties of the tissue.

Although OA is a common disease, an accurate diagnosis of early OA remains elusive in clinical practice because the changes leading to it precede significantly the OA symptoms. Therefore, a sensitive technique for detecting the early structural and functional changes in OA cartilage would be valuable for monitoring disease progression and for evaluating the efficacy of treatment. Clinically, conventional radiography has been the standard test for assessing joint space narrowing, yet its sensitivity for detecting early stage OA is limited. ${ }^{34}$ In the past decade, magnetic resonance imaging (MRI), which can visualise changes in soft tissues and report them quantitatively, has emerged as a possible diagnostic technique to assess cartilage degeneration and to detect early signs of OA non-destructively. ${ }^{6}$ Recent studies have shown that MRI can detect cartilage morphological abnormalities and measure changes in cartilage volume. ${ }^{7}$ However, low resolution and artefacts such as partial volume averaging remain obstacles in monitoring microstructural changes of cartilage degeneration in clinical MRI.

Microscopic MRI ( $\mu$ MRI) has been recently used to study cartilage structure and its topographical variations in vitro. Specifically, the change of collagen fibrillar orientations in cartilage can cause variations in MR intensity images in three histological zones in cartilage (superficial, transitional, and deep), the so-called laminar appearance or "magic angle" effect in cartilage..$^{10}$ The origin of such artefactual appearance of cartilage in MRI has recently been confirmed to be the $T_{2}$ relaxation modulated by nuclear dipolar interaction. ${ }^{11}$ In addition, $T_{2}$ relaxation anisotropy in $\mu$ MRI has been shown to reveal the properties of individual histological zones non-destructively, just as such subtissue zones can be visualised optically based on collagen fibre orientation in polarised light microscopy (PLM). ${ }^{12}$

Surgical transection of the anterior cruciate ligament (ACL) in dogs is a useful model for studying the early phases of OA in knees. This well characterised animal model of posttraumatic OA creates joint instability that eventually leads to end stage cartilage loss, and mimics the changes of natural $\mathrm{OA}$ in both dogs and humans. ${ }^{13}$ We propose to study early changes in cartilage microstructure in experimental OA using high resolution $\mu$ MRI. Specifically, because both the $T_{2}$ anisotropy technique in $\mu \mathrm{MRI}$ and the angle imaging technique in PLM are sensitive to the orientation of the

Abbreviations: $\mathrm{ACL}$, anterior cruciate ligament; $\mu \mathrm{MRI}$, microscopic magnetic resonance imaging; OA, osteoarthritis; PLM, polarised light microscopy; ROI, region of interest 
collagen fibril structure in cartilage, ${ }^{12}{ }^{14}$ we suggest that the early OA tissue will have some distinct changes at ultrastructural and morphological levels related to the alternation to the collagen matrix, and that such changes can be detected by the combined $\mu$ MRI-PLM experiment at microscopic resolution. Although the resolution in this microscopic study is currently not possible in clinical MRI, successful work at microscopic resolution can serve as an inducement, for the MRI researchers as well as the instrument manufacturers to improve the clinical resolution and to investigate a resolution limit below which such a detection is not possible (owing to various artefacts and a volume averaging process among different structures within a single voxel).

\section{METHODS}

\section{Animal model and samples preparation}

Three skeletally mature dogs, weighing $29-32 \mathrm{~kg}$, underwent anterior (cranial) cruciate ligament (ACL) transection in one knee joint. The contralateral knee served as an unoperated internal control. ${ }^{15}$ Animals were handled according to the protocols approved by the Institutional Review Boards. The animals were killed 12 weeks postoperatively for an unrelated study. The proximal tibias of the control and OA limb were collected from each animal. The joints were shipped overnight in an insulated container containing ice packs. Specimens from two pairs of joints were immediately excised and stored at $4^{\circ} \mathrm{C}$; the other pair of joints was stored at $-80^{\circ} \mathrm{C}$ until ready for experiments.

The meniscus was dissected and three full-thickness, rectangular $(\sim 2 \times 2 \times 12 \mathrm{~mm})$ osteochondral specimens were cut from the medial tibial plateau as shown in fig 1 . The total number of specimens in this study is 18 (nine controls and nine OA). The specimens were bathed in freshly prepared physiological saline with added Protease Inhibitor cocktail (P2714; Sigma Co, St Louis, MO, USA). Twelve specimens from two animals were sealed in precision nuclear magnetic resonance (NMR) tubes with an internal diameter of $2.34 \mathrm{~mm}$ and six specimens from one animal were sealed in precision NMR tubes with an internal diameter of $4.34 \mathrm{~mm}$ (Wilmad Glass, Buena, NJ, USA).

\section{$\mu M R I$ experiments}

Quantitative two dimensional $\mathrm{T}_{2}$ relaxation maps were made with a Bruker AMX 300 spectrometer equipped with a 7 T/89 $\mathrm{mm}$ magnet and microimaging accessory (Bruker

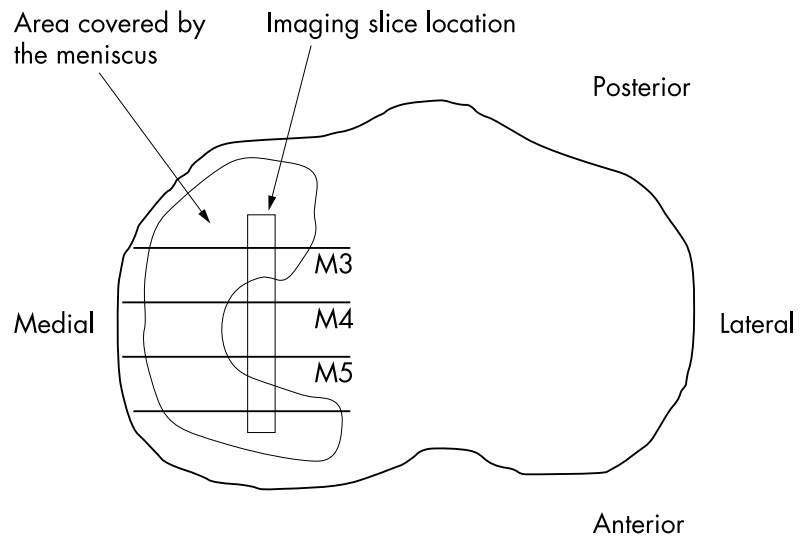

Figure 1 A schematic view of the tibia surface from canine knee joint with the location of specimens in the imaging experiments indicated on the central area of the tibia. The imaging slice location of $M 4$ is not covered by the meniscus, whereas M5 and M3 imaging slice locations are at the margin of the meniscus.
Instruments, Billerica, MA) and two ( 3 and $5 \mathrm{~mm}$ diameter) solenoid coils. All the experiments were conducted at about $25^{\circ} \mathrm{C}$ room temperature in the magnet, using a $\mathrm{T}_{2}$ weighted, spin echo imaging sequence. The echo time of the imaging segment was $11.6 \mathrm{~ms}$, the echo time of the leading contrast segment had four increments: (1.6, 16, 30.4, and $60.8 \mathrm{~ms}$ ), and the repetition time of the imaging experiment was 2 seconds. A mono-exponential fit model was used to calculate $T_{2}$ relaxation pixel by pixel. The in-plane resolution across the depth of the cartilage tissue was $13.7 \mu \mathrm{m}$ and $23.1 \mu \mathrm{m}$ for the $3 \mathrm{~mm}$ coil and the $5 \mathrm{~mm}$ coil, respectively. Other experimental details have been described earlier. ${ }^{14}$

After loading the specimen into the $\mu$ MRI probe and before acquiring two dimensional $\mathrm{T}_{2}$ maps, two pilot images were obtained: a sagittal pilot and a coronal pilot. The sagittal pilot was used to align the articular surface of cartilage to the nominal angle; and the coronal pilot was used to position the $1 \mathrm{~mm}$ MRI slice to minimise the contribution of saline signal at the articular surface boundary. A custom in situ samplerotating device was used to obtain four $T_{2}$ weighted $M R$ images at each of the four orientations $\left(0^{\circ}, 36^{\circ}, 55^{\circ}\right.$, and $\left.90^{\circ}\right)$ for each specimen. $\mathrm{T}_{2}$ relaxation was then fitted using the program ParaVision from Bruker Instrument with a 3\% threshold.

\section{Mechanical testing}

After $\mu \mathrm{MRI}$, cartilage-bone specimens were tested in compression to determine the mechanical properties of cartilage by measuring the tangent Young's modulus for each sample. Briefly, a flat, $2 \mathrm{~mm}$ diameter, non-porous, stainless steel indentor was driven into the cartilage using an EnduraTEC 3200 machine equipped with WinTest software (EnduraTEC, Minnetonka, MN, USA) and a $100 \mathrm{lb}$ (45 kg) load cell (Sensotec, Columbus, OH, USA). The specimens were bathed in saline and three local tests were performed for each specimen, with a 30 minute waiting period between each test. For each test, a displacement-control ramp $(0.005 \mathrm{~mm} /$ s) was used keeping the strain rate within the physiological range $(\sim 10-15 \%)$. Subsequently, a stress-strain plot was generated from each test and the slope of the linear portion of each plot was calculated to determine the tangent Young's modulus. ${ }^{16}{ }^{17}$ Cartilage thickness, measured from $\mu$ MRI, was used to calculate strain values. The reported Young's modulus of each specimen is the average of three local tests.

\section{Histology and PLM experiments}

All specimens that had been examined by $\mu$ MRI and mechanical indentation were then prepared for histology. Each specimen was cut at approximately the same MRI slice location and the cartilage-bone plug was embedded in OCT medium and snap frozen in liquid nitrogen and stored at $-80^{\circ} \mathrm{C}$ until sectioning. Ten to 20 frozen sections $(14 \mu \mathrm{m}$ thickness) were prepared from each specimen in cryostat microtome and were examined unstained. The histology and $\mu$ MRI of frozen sections are more comparable because there is less tissue shrinkage and fewer alterations of the collagen from conventional processing. The use of unstained sections ensures a more accurate measurement of the tissue properties because staining can enhance (and hence distort) the contrast of the matrix components. To generate quantitative optical images from the histological sections, a Leica DMRX Microscope (Leica, Microsystems Wetzlar GmbH, Wetzlar, Germany) equipped with a liquid crystal compensator and a CCD camera was used. ${ }^{12}$ The final two dimensional images were the optical retardance and the average angular orientation of the collagen fibres in cartilage tissue, with a pixel resolution of $2.72 \mu \mathrm{m}$ at $\times 5$ magnification. 


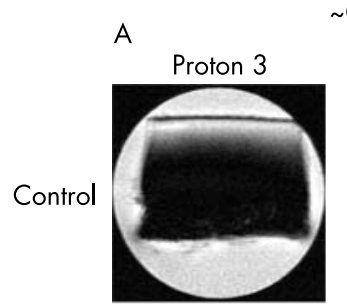

C

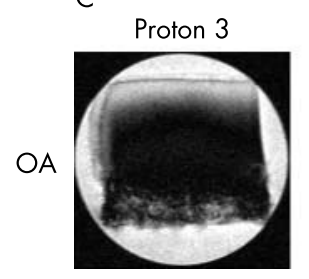

$\sim 0^{\circ}$
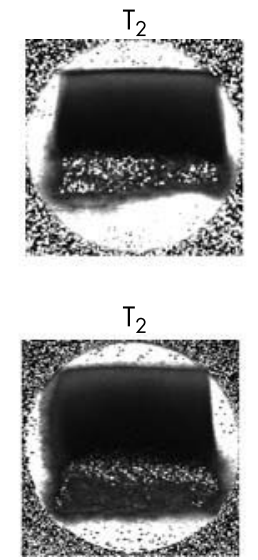

B

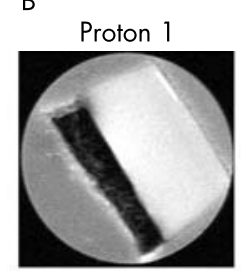

D

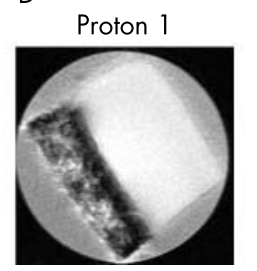

$\sim 55^{\circ}$
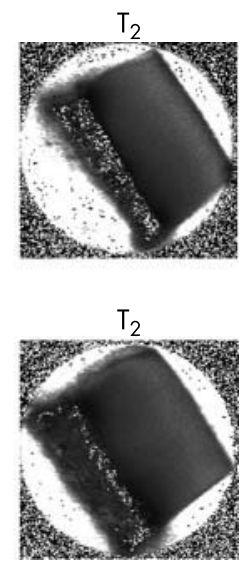

Figure 2 From the upper left: (A) proton 3 and $\mathrm{T}_{2}$ image of a control tibia specimen at $\sim 0^{\circ} ;(\mathrm{B})$ proton 1 and $\mathrm{T}_{2}$ image of the same control tibia specimen at $\sim 55^{\circ}$; (C) proton 3 and $\mathrm{T}_{2}$ image of an OA tibia specimen at $\sim 0^{\circ}$; (D) proton 1 and $\mathrm{T}_{2}$ image of the same OA tibia specimen at $\sim 55^{\circ}$. The images shown above are specimens from a matched set from the same animal with a resolution of $13.7 \mu \mathrm{m}$. Each specimen was imaged with four different $\mathrm{T}_{2}$ weightings. The angle is defined as the angle between the normal to the articular surface of cartilage and the direction of the magnetic field $\left(B_{0}\right)$.

\section{Data analysis and statistical interpretation}

To compare fairly the data from different sections/specimens, one dimensional profiles through the depth of the cartilage tissue were extracted from all two dimensional images. From $\mu$ MRI experiments, a 20 pixel width region of interest (ROI) was averaged from the middle of two dimensional $\mathrm{T}_{2}$ images using commercially available software IPLab (Scanalytics, Fairfax, VA, USA). The images were rotated on the computer to align the cartilage articular surfaces as needed. In MRI images, cartilage thickness was measured using the first $T_{2}$ weighted proton image of each specimen, and five measurements from each specimen were averaged at different locations. In PLM images, a 100 pixel width ROI, from both the retardance and the angle images, was averaged at the same location of ROI used in the $T_{2}$ images from each histological section. A $5 \times 5$ block average filter was used to smooth the one dimensional PLM profiles, which adjusted the in-plane PLM resolution to $13.6 \mu \mathrm{m}$ along the tissue depth, approximately equal to the individual $\mu \mathrm{MRI}$ pixel size $(13.7 \mu \mathrm{m})$. The retardance and the angle profiles from each histological section were then averaged among all histological sections.

Owing to the noticeable (and $\mathrm{known}^{18}$ ) variations in cartilage thickness across the tibia, the depth of each one dimensional profile was normalised to 1 , where the relative depth 0 represents the articular surface and the relative depth 1 represents the boundary between cartilage tissue and bone. This normalisation facilitates direct comparisons among different MRI resolutions, different imaging modalities ( $\mu$ MRI and PLM), and different tibial regions. Each one dimensional profile was curve fitted with cubic spline in KaleidaGraph, and an average of 70 and 40 points along the cartilage depth was obtained from the $13.7 \mu \mathrm{m}$ resolution profiles and the $23.1 \mu \mathrm{m}$ resolution profiles, respectively. Repeated measures, two way analysis of variance was used to analyse all parameters measured from MRI, PLM, and mechanical testing (Prism by GraphPad Software, San Diego, CA, USA). We considered two main factors that might influence the interpretation of this study: topographical location and OA model. If the interaction between these factors was significant, then we would ignore the significance of each factor because in such a case it is hard to interpret the significance of each individual factor. If the interaction was not significant, then the significance of each individual factor, measured independently from the other factor, would be taken into consideration in the resulting interpretation. ${ }^{19}$ For each measurement at a specific topographical location, a paired Student's $t$ test was used to test the significance of differences between OA and control pairs.

\section{RESULTS}

\section{Morphology observations}

In all control knees, the meniscus was intact and the surfaces of the femoral and tibial articular cartilage were normal. In all OA knees, the medial meniscus was partly torn (in one a large circumferential tear), the medial femoral condyles showed partial thickness cartilage erosions, and there were multiple osteophytes around the joint periphery. There were no signs of gross cartilage surface fibrillation on the tibial surfaces from OA joints. The articular cartilage of the OA joints appeared more opaque and thicker than the cartilage of control joints, which appeared thinner and translucent.

Figure 2 shows the proton images and the calculated $\mathrm{T}_{2}$ images of a control specimen and an OA specimen (from the M5 location of one pair) at two different orientations: $0^{\circ}$ and $55^{\circ}$. At $0^{\circ}$, all $\mathrm{OA}$ and control specimens had a similar laminar appearance. Upon close examination, the low intensity band underneath the articular surface, which corresponds to the superficial zone, was thinner (fig 2C) and the cartilage total thickness was greater in OA specimens. (This visual observation was confirmed later in quantitative measurements in $\mu$ MRI and PLM.) In addition, the control specimens and OA specimens showed the same homogeneous appearance at the magic angle (figs $2 \mathrm{~B}$ and $\mathrm{D}$ ).

\section{Quantitative correlation between $\mu$ MRI and PLM}

To assess the integrity of the collagen fibril network, one dimensional profiles from the corresponding two dimensional $\mu$ MRI or PLM images of $\mathrm{T}_{2}$ relaxation, optical retardation, and fibril angle were evaluated. Figure 3 shows a representative set of one dimensional MRI and PLM profiles from a single specimen. Using the quantitative criteria established and validated previously, ${ }^{12}{ }^{20}$ all $\mu$ MRI images were subdivided structurally into histological zones based on the $\mathrm{T}_{2}$ profile at $0^{\circ}$ and all PLM images were subsectioned into histological zones based on the angle profile. Table 1 summarises the results from all specimens. 


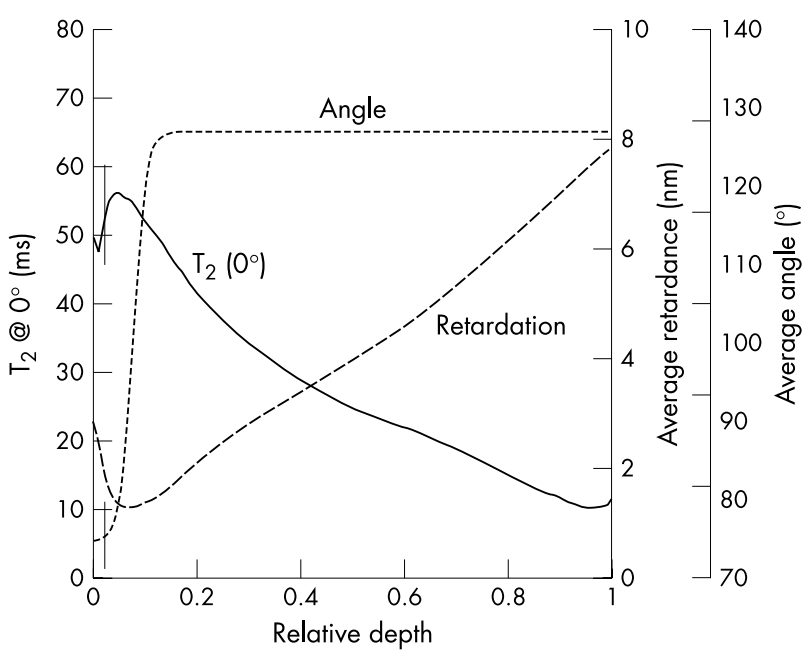

Figure 3 At the left $y$ axis: $T_{2}$ profile at $\sim 0^{\circ}$ versus the relative depth extracted from a 20 pixel column from the middle of an OA specimen with $13.7 \mu \mathrm{m}$ in-depth resolution (solid line). At the right y axis: average optical retardation (dashed line) and average collagen orientation angle (dotted line) were plotted versus the relative depth of the same OA specimen with $13.6 \mu \mathrm{m}$ in-depth resolution. The vertical lines correspond to the determined border between the superficial and the transitional zone.

For each orientation, all individual $\mathrm{T}_{2}$ profiles have essentially the same curve pattern, which indicates no severe damage of fibril structure in OA specimens. This overall observation in $\mu$ MRI was confirmed optically by the consistency in the curve patterns between the control and OA pair in PLM measurement. A comparison of each pair of $\mathrm{T}_{2}$ profiles (at the same orientation from the matched sets of a normal knee and an OA knee from the same animal), however, showed some changes in the macromolecular structure due to surgical intervention (fig 4A). Even though both $T_{2}$ profiles have the same curve pattern that contains one major peak, we observed a significant shift in the depth of maximum $\mathrm{T}_{2}$ towards the articular surface in OA specimens. Interestingly, this shift of $\mathrm{T}_{2}$ peak was absent in all specimens that were completely uncovered by the meniscus (M4) and took place in other specimens from the central tibia (M3 and M5, see fig 1). In addition to the peak shift, we also noticed an increase in the average value of $\mathrm{T}_{2}$ in the superficial zone in two locations (M3 and M5) owing to the introduction of $\mathrm{OA}$, although this increase in $\mathrm{T}_{2}$ values in the superficial zone was not statistically significant (see table 2 ). These $\mu$ MRI observations were confirmed by the one dimensional profile analysis in PLM: the minimum retardance also exhibits a similar shift towards the articular surface in the OA specimens when compared with control specimens from similar topographical locations (fig 4B).

Figure 5 shows the correlation between the depths of maximum $\mathrm{T}_{2}$ from $\mu \mathrm{MRI}$ and minimum retardance from PLM for each topographical location $(n=3$ for each of the M3, M4, and M5 locations, see fig 1). Although the OA specimens at the M4 location do not exhibit a shift in the depths of maximum $\mathrm{T}_{2}$ and minimum retardance towards the articular surface, M3 and M5 have a 36\% and $21 \%$ shift in the depth, respectively.

To examine whether $T_{2}$ values can be used as a sensitive marker of tissue degeneration, we compared $T_{2}$ values of controls and OA model specimens. To simplify the comparison and to mimic the low resolution in clinical MRI, the $\mathrm{T}_{2}$ profiles over the entire depth were averaged to give "bulk" values, and compared at all orientations $\left(0^{\circ}, 36^{\circ}, 55^{\circ}\right.$, and $\left.90^{\circ}\right)$ for each topographical location (M3, M4, and M5). Figure 6 shows the results obtained-the closer the point is to the

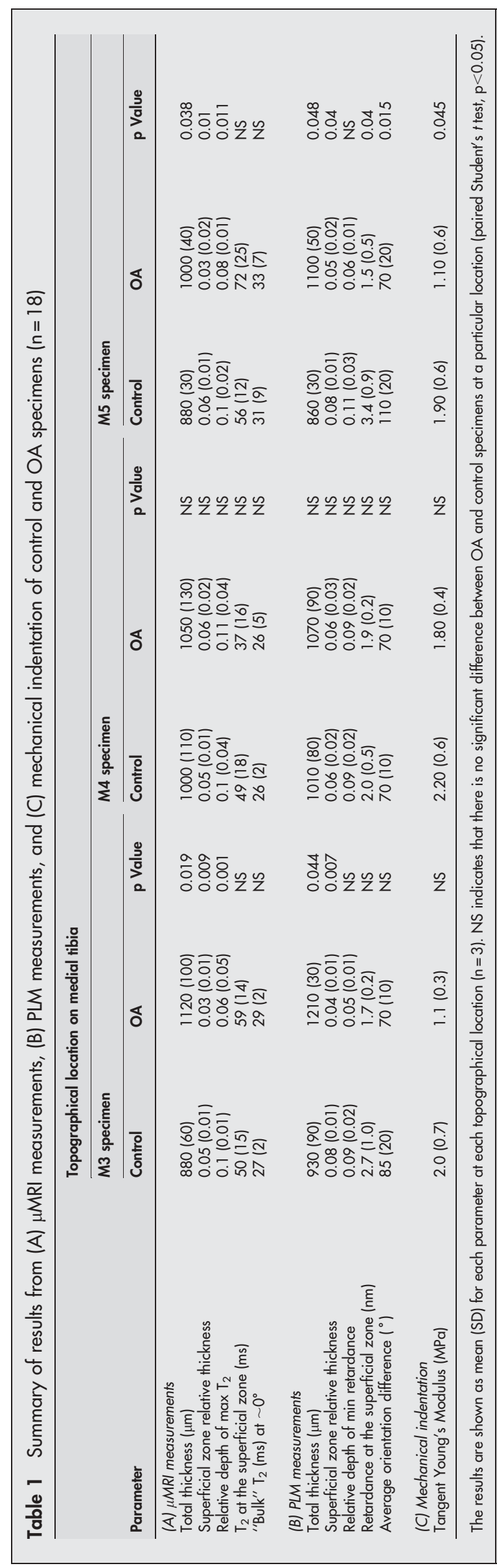



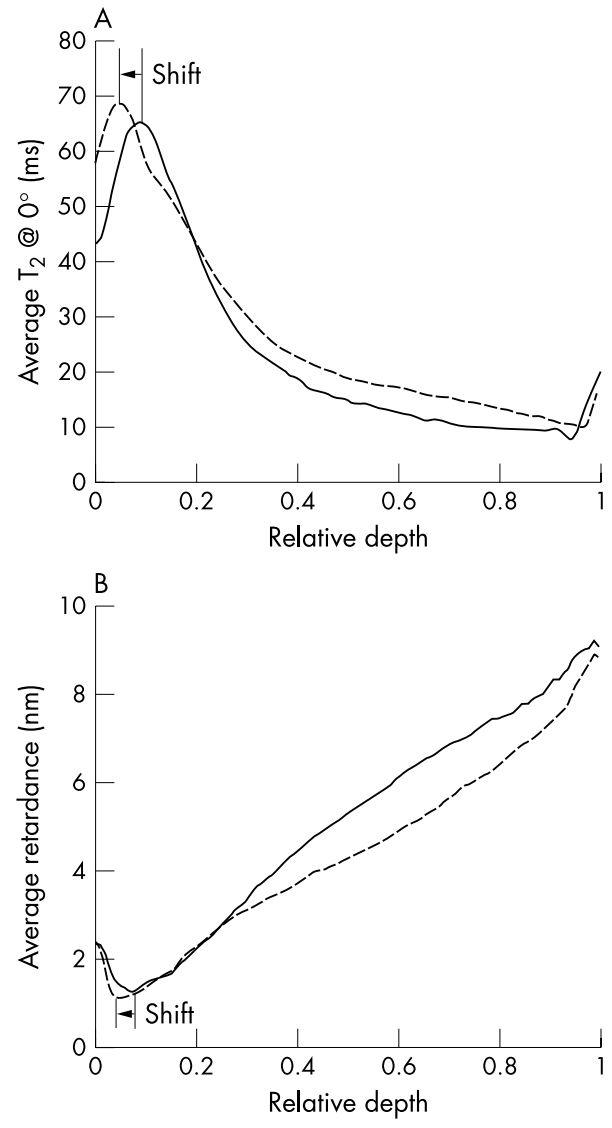

Figure 4 (A) Average $T_{2}$ profiles of $O A$ and control specimens at $\sim 0^{\circ}$ from the M3 location $(n=3)$ versus the relative depth. Notice the change in the thickness of the surface zone and subsequently the shift of the relative depth of maximum $\mathrm{T}_{2}$ between control specimens (solid line) and $O A$ specimens (dashed line). (B) Average retardance profiles of $O A$ and control specimens from the $M 3$ location $(n=3)$ versus the relative depth. Notice the shift of the relative depth of minimum retardance between control specimens and OA specimens.

Table 2 Repeated measures, two way analysis of variance of the results from (A) $\mu M R I$ measurements, (B) PLM measurements, and (C) mechanical indentation of control and OA specimens $(n=18)$

\begin{tabular}{|c|c|c|c|}
\hline Parameter & $\begin{array}{l}\text { Interaction } \\
\text { (p value) }\end{array}$ & $\begin{array}{l}\text { Location } \\
\text { (p value) }\end{array}$ & $\begin{array}{l}\text { Disease } \\
\text { (p value) }\end{array}$ \\
\hline \multicolumn{4}{|l|}{ (A) $\mu \mathrm{MRI}$ measurements } \\
\hline Total thickness & $<0.05$ & * & * \\
\hline Relative thickness of superficial zone & NS & NS & $<0.01$ \\
\hline Relative depth of $\max T_{2}$ & $<0.05$ & * & * \\
\hline $\mathrm{T}_{2}$ at the superficial zone & NS & NS & NS \\
\hline "Bulk" $\mathrm{T}_{2}$ at $\sim 0^{\circ}$ & NS & NS & NS \\
\hline \multicolumn{4}{|l|}{ (b) PLM measurements } \\
\hline Total thickness & $<0.05$ & * & * \\
\hline Relative thickness of superficial zone & & NS & $<0.05$ \\
\hline Relative depth of min retardance & & NS & $<0.05$ \\
\hline Retardance at the superficial zone & & NS & $<0.01$ \\
\hline Average orientation difference $\left({ }^{\circ}\right)$ & $<0.01$ & * & * \\
\hline \multicolumn{4}{|l|}{ (C) Mechanical indentation } \\
\hline Tangent Young's modulus & NS & NS & $<0.01$ \\
\hline \multicolumn{4}{|c|}{$\begin{array}{l}\text { NS indicates not statistically significant and the star symbol }\left(^{*}\right) \text { indicates } \\
\text { that the } p \text { value of a single factor is ignored if the interaction is significant } \\
(p<0.05) \text {. }\end{array}$} \\
\hline
\end{tabular}

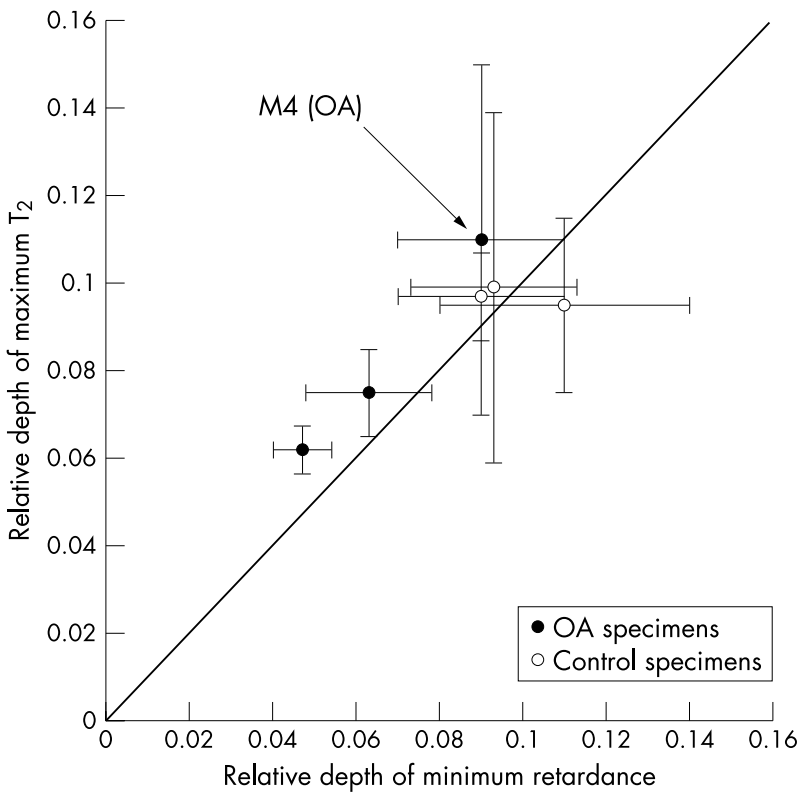

Figure 5 The trend of the relative depth of minimum retardance and the relative depth of maximum $\mathrm{T}_{2}$ at $\sim 0^{\circ}$. Each point corresponds to the mean of relative depth at a specific location $(M 3, M 4$, and $M 5, n=3)$.

solid diagonal line, the better the agreement between the OA $\mathrm{T}_{2}$ and control $\mathrm{T}_{2}$. Several important observations can be made. Firstly, $\mathrm{T}_{2}$ in the OA specimens is in general higher than that in the control specimens, because nearly all data points are below the solid diagonal line. However, we did not see a significant change in $\mathrm{OA} \mathrm{T}_{2}$ at $0^{\circ}$. Secondly, $\mathrm{T}_{2}$ values of M4 specimens at all orientations show less variation between OA and control than the M3 and M5 locations, in support of other results in this report demonstrating the topographical variations. Thirdly, $\mathrm{T}_{2}$ measured at $0^{\circ}$ varied least between $\mathrm{OA}$

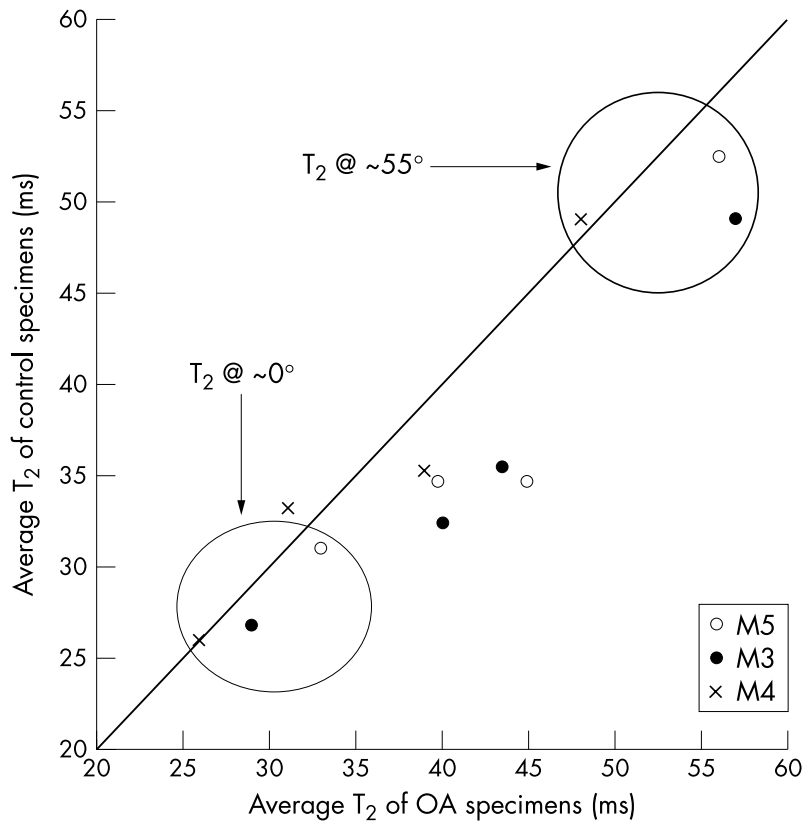

Figure 6 Average "bulk" $T_{2}$ measurements for each topographical location $(n=3)$ at four different orientations $\left(0^{\circ}, 36^{\circ}, 55^{\circ}\right.$, and $\left.90^{\circ}\right)$. Note that $\mathrm{T}_{2}$ at $\sim 0^{\circ}$ shows the least variation between control and $\mathrm{OA}$ specimens. 
and controls, regardless of the topographical location of the specimen, whereas $T_{2}$ varied most when specimens were oriented at the magic angle.

Although all individual $\mathrm{T}_{2}$ profiles have essentially the same curve pattern, some subtle effects of OA on different histological zones can be seen across the thickness of the tissue. Figure 7 shows a comparison of the OA and control specimens using the averaged $\mathrm{T}_{2}$ profile, across the central tibia surface, of all three specimens (M3, M4, and M5) from the same tibia. Even averaged, a shift in the depth of peak $\mathrm{T}_{2}$ can still be noticed in the OA joint in comparison with the control joint. Most remarkably, the deep zone of cartilage tissue $(\sim 0.2-1$ relative depth) exhibits greater variations in $\mathrm{T}_{2}$ values (represented by higher standard deviations along the relative depth) in the OA joint than in the control joint. Because each of the $T_{2}$ profiles averages three topographically different sites in the tibia, the smaller standard deviations in the control joints imply that the $\mathrm{T}_{2}$ characteristics in the deep tissue were almost the same among the control specimens from topographically different locations. In contrast, the larger standard deviations in the OA joints imply that the $\mathrm{T}_{2}$ characteristics in the deep tissue were noticeably different among the OA specimens from the topographically different locations, signalling the important site variations at the early stages of the diseases.

One of the early signs of $\mathrm{OA}$ is the increase in water content and the slight initial changes in cartilage total thickness. Figure 8 shows the cartilage total thickness of all 18 specimens from MRI and PLM measurements. Close proximity to the line of identity indicates a good correlation between the two measurements (correlation coefficient, $r=0.78, \mathrm{p}<0.001)$; the separation of $\mathrm{OA}$ and control values measures the increase in cartilage thickness due to surgical treatment. The scatter within each cluster may be due to variation in thickness at the three different site locationsthe thickness here was not normalised.

Table 1 summarises all the results from $\mu$ MRI and PLM imaging measurements as well as the mechanical testing of all specimens. Several conclusions can be drawn from the

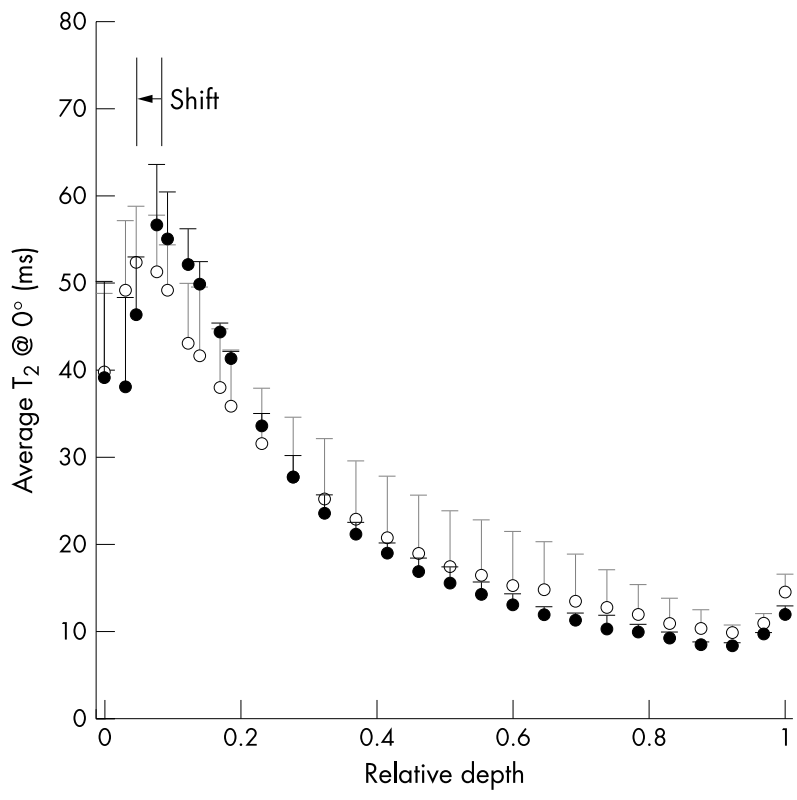

Figure 7 Average $\mathrm{T}_{2}$ profile at $\sim 0^{\circ}$ from the three adjacent specimens (M3, M4, and M5) from a normal tibia and an OA tibia from a matched pair, with $13.7 \mu \mathrm{m}$ resolution. The error bars correspond to the variations in $\mathrm{T}_{2}$ among the adjacent specimens. (For clarity, only half of the points were plotted.)

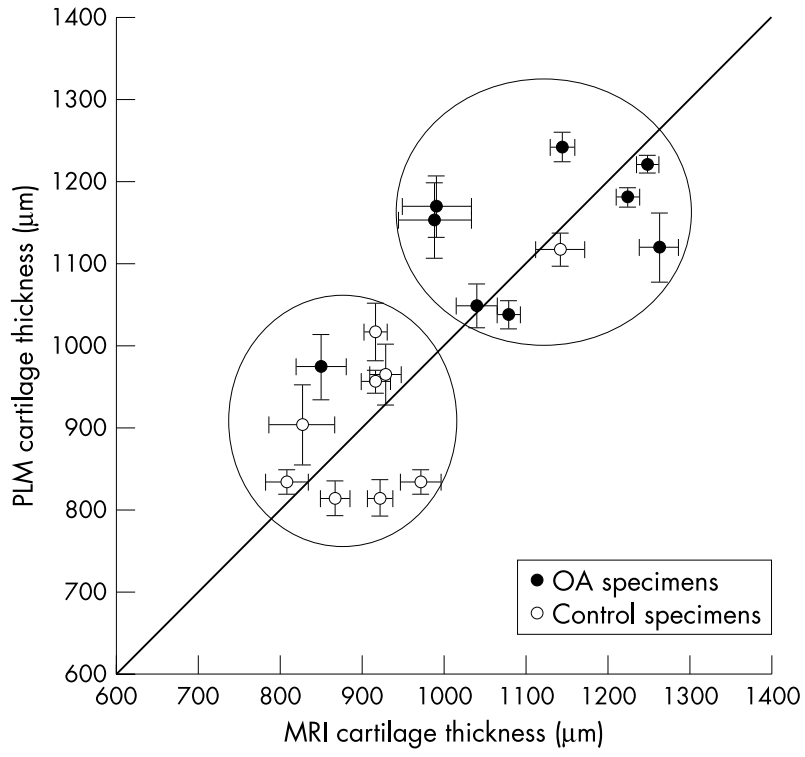

Figure 8 Total thickness of cartilage specimens from both MRI images and PLM images $(n=18)$. The linear correlation coefficient between MRI measurements and PLM measurements is 0.78 .

table. (1) An increase in the cartilage total thickness was seen in all OA specimens compared with the control specimens from both the $\mu$ MRI and PLM results. The increase was $(28.7 \%, 5.4 \%$, and $30.4 \%)$ in PLM and $(14.5 \%, 4.5 \%$, and $27.4 \%$ ) in $\mu$ MRI for specimens (M5, M4, and M3), respectively. However, the increase was not significant in the central specimen M4 $(\mathrm{p}<0.05)$. (2) Only the M5 and M3 specimens showed a significant reduction in the thickness of the superficial zone of OA joints. The change from $\mu$ MRI results was $(-38.2 \%,-37.3 \%)$ and from the PLM results was $(-44.6 \%,-48 \%)$ for (M5, M3), respectively. (3) A decrease in the retardance values at the superficial zone was seen in all OA specimens, although it was only significant in one location (M5). Similarly, an increase in $\mathrm{T}_{2}$ value at the superficial zone was detected in OA specimens from M5 and M3 locations; however, it was not statistically significant in either. A change in the fibre orientation difference between the superficial zone and the deep zone was detected in OA specimens from M5 and M3 locations; however, it was only significant in M5. (4) "Bulk" $\mathrm{T}_{2}$ measurements (the averaged $\mathrm{T}_{2}$ value from the entire tissue depth) did not show any significant change in early degenerative state at a given topographical location. (5) The measured mechanical strength shown in table 1 indicates a softened tissue for all specimens as a result of the introduction of OA to the joints. The tangent Young's modulus was reduced by $(40.2 \%, 16.3 \%$, and $43.9 \%$ ) for specimen (M5, M4, and M3), respectively. Note that the M4 specimens had the smallest reduction in the mechanical modulus, in excellent agreement with the observed changes at this particular site by $\mu$ MRI and PLM.

In addition, to examine each group of control and OA specimens at a specific topographical location individually, a broader statistical analysis of all measured parameters was performed to consider the topographical variations in tibia and the morphological differences between weightbearing and non-weightbearing areas in cartilage. Table 2 shows the significance of the joint location and OA model, and the interaction of both, for each measured parameter from all 18 specimens $(p<0.05)$. Several conclusions can be drawn from this set of statistical analyses. (1) The cartilage total thickness had changed significantly owing to the interaction between the OA model and the specimen location from both the MRI 
and PLM results. (2) By comparison, no interaction was detected for superficial zone thickness. (3) The relative depth of maximum $\mathrm{T}_{2}$ also interacted significantly with joint location and OA model, whereas the relative depth of minimum retardance showed only a change due to the OA model. (4) We found no significant change in $\mathrm{T}_{2}$ value in the superficial zone or in bulk $\mathrm{T}_{2}$; however, the retardance value changed significantly owing to the introduction of experimental OA. (5) Finally, mechanical testing showed a significantly reduced value in tissue mechanical properties in the OA specimens, with no significant influence from the location of the central specimens at the tibia surface.

\section{DISCUSSION AND CONCLUSIONS}

Few sensitive tests are currently available to assess the early changes in osteoarthritic cartilage non-destructively. The "disintegration" of the collagen fibrillar network is thought by many to be the initiating event in the focal erosion of articular cartilage in OA. ${ }^{21}$ In this $\mu$ MRI and PLM imaging study, we investigated the ultrastructural changes in articular cartilage in an animal OA model. Using the depth dependent fibrillar structure as a basis for subdividing cartilage tissue into individual histological zones, we show that $\mu \mathrm{MRI}$ and PLM can monitor the microscopic changes in degenerative cartilage according to depth. In particular, ${ }^{12}$ the $T_{2}$ relaxation profile in articular cartilage has a strong depth dependent anisotropy; the angle profile in tissue represents the fibre orientation along the tissue depth; and the optical retardance profile represents the orientational coherence of the collagen fibrils within a finite image volume. For healthy cartilage, ${ }^{12}$ the $T_{2}$ profile, when measured at $0^{\circ}$, is characterised by an asymmetric bell shaped curve, with its maximum indicating the most random location in the tissue; the angle profile typically follows a hyperbolic tangent curve that represents a clear transition of the overall orientation of the fibres along the cartilage depth (three zones); and the optical retardation profile is characterised by an inverted bell shaped curve, with its minimum indicating the least ordered region in tissue. The sensitivity of both $\mathrm{T}_{2}$ anisotropy in $\mu \mathrm{MRI}$ and angle imaging in PLM to fibril orientation make it possible to detect any structural change in cartilage.

\section{Changes in morphological properties in cartilage during the early stages of $O A$}

The morphological observation of the joints used in this study has confirmed that these cruciate transected joints are consistent with joints in the early "hypertrophic" phase of this experimental OA. ${ }^{22}$ The swelling of cartilage is one of the initial signs of the structural disruption associated with an increase of water content in a degenerative state. In this high resolution study, the total thickness of OA cartilage increased significantly in two locations of the central tibia, in good agreement with published data. ${ }^{23-26}$ However, the broader statistical analysis (table 2) indicates that total thickness alone is not a reliable measure for detection of OA because the topographical variation over the joint surface interacts with the increase in OA model. By comparison, change in the superficial zone thickness can be used as a reliable indicator of early OA, because this measure is influenced little by the topographical variation, if it exists.

The consistent MRI appearance of cartilage laminae from all OA and control specimens in this study shows that in the early stages of OA, the collagen fibril network is largely intact and does not suffer severe disruption, at the morphological scale, to its structure of three histological zones. This apparently "normal" MRI appearance of cartilage tissue at early disease stages attests to the need for a high resolution quantitative approach in clinical imaging, and can explain some apparent inconsistency in published reports.
Comparing degenerative cartilage between in vitro imaging and clinical imaging, Rubenstein et al showed that MRI of advanced disease of human cartilage was marked with fraying of the articular surface and loss of trilaminar appearance, and that as pixel size increases, some of the signs of degenerative cartilage (such as fibrillated surface) gradually disappear from the MRI appearance. ${ }^{27}$ Future studies are needed to establish a resolution limit above which this detection of early disease is no longer reliable.

We also observed in this study several topographical changes of early OA on medial tibial cartilage: although there are significant changes in M3 and M5 specimens, there are no observed changes in the in-between "central" specimen (M4) as a result of the introduction of OA. In addition, the results in fig 7 show that the most visible difference in normal tibia is at the surface, because the $\mathrm{T}_{2}$ characteristics in the deep tissue were almost the same among the control specimens from topographically different locations. However, the disease may have different impacts on the tissue topographically, because the $\mathrm{T}_{2}$ characteristics in the deep tissue were noticeably different among the OA specimens from topographically different locations. Early electron microscopy studies of tibial plateau have shown that the collagen organisation is not the same across the tibia plateau and the variations in mechanical and structural properties might be related to the weightbearing regions. ${ }^{28}{ }^{29}$ The results in this report, together with published data, suggest that some areas in tibia covered by meniscus will show early signs of degeneration before it is evident in other areas of the joint.

\section{Changes in physical properties in cartilage during the early stages of $O A$}

In MRI studies, cartilage is known to have a strong $\mathrm{T}_{2}$ anisotropy, originating from the influence of the collagen orientation on nuclear dipolar interaction. ${ }^{9}$ Because the structure of the collagen matrix varies along the tissue depth (the histological zones), the $\mathrm{T}_{2}$ anisotropy in articular cartilage is strongly dependent on depth (the MRI laminae). Consequently, it is reasonable to suggest that any change in the ultrastructural organisation of the collagen fibril network and interactions among collagen/proteoglycans/water due to the diseases would influence the characteristics of $\mathrm{T}_{2}$ anisotropy in cartilage.

The shift of the maximum $\mathrm{T}_{2}$ depth, which corresponds indirectly with the most random region of the collagen fibril network based on the mobility of water molecules, ${ }^{20}$ towards the articular surface supports our understanding that the collagen fibres in the superficial zone were disrupted in fibril orientation at the early OA stages. More specifically, this shift indicates that some surface fibres, which ran more parallel to the articular surface in healthy tissue, became more random in lesion. This $\mu$ MRI conclusion is supported by our observation of a similar shift of the minimum retardance depth, which corresponds directly with the most random region of the collagen fibril network. Statistical analysis (table 2) shows that both the topographical location and the $\mathrm{OA}$ model influence the maximum $\mathrm{T}_{2}$ peak, whereas the depth of the minimum retardation is influenced by the OA model alone, probably the consequence of a higher signal to noise ratio and finer optical resolution in PLM. Our results are in agreement with a published electron microscope study of canine lateral femoral condyle cartilage, where the collagen fibrils from the superficial zone were predominately parallel to the surface, but "exhibited a 'wavy' appearance and occasional fibrils ran obliquely" after 8 weeks of ACL sectioning. ${ }^{30}$

A question related to the observation of the $T_{2}$ peak shift in OA specimens is: can the value of $\mathrm{T}_{2}$ be used as a marker for the degeneration of the tissue? ? $^{31-34}$ Clearly, many 
experimental factors can significantly affect the measured values of $\mathrm{T}_{2}$ in cartilage. Apart from a number of experimental MRI factors such as the pulse sequence and imaging timings, three biological factors also make the establishment of a "normal" $\mathrm{T}_{2}$ value in cartilage difficult. They are the orientational dependency of the tissue in a magnet, the topographical variation of the specimen on a joint surface, and the depth dependency of the $T_{2}$ value at different histological zones. In particular, the fact that $\mathrm{T}_{2}$ at $0^{\circ}$ shows the least enhancement in OA specimens demonstrates the importance of orientational dependency of a specimen in clinical setting: if one wants to use $\mathrm{T}_{2}$ enhancement to monitor early tissue degradation, then the specimen needs to be oriented towards the magic angle $\left(\sim 55^{\circ}\right)$. This is because the influence of nuclear dipolar interactions on $\mathrm{T}_{2}$ at $0^{\circ}$ may be significant, consequently overshadowing the influence of the early $\mathrm{OA}$ on $\mathrm{T}_{2}$. Our results show that even a small change in orientation ( such as at $36^{\circ}$ or $90^{\circ}$ ) could help in "turning off" the influence of $\mathrm{T}_{2}$ anisotropy to better observe OA changes.

In conclusion, this study of the microstructural changes in degenerative cartilage, which to our knowledge is the highest resolution imaging study of lesioned cartilage in tibia, is unique in several aspects. Firstly, the high resolution $\mu$ MRI provides us with a critical advantage to detect small disruption in a local area. The significant changes detected in the superficial zone and the depth of the random location in cartilage in this study are less than the size of one pixel in routine clinical MRI, which will not be noticeable otherwise. Secondly, our $\mu$ MRI results correlate well with the results from the "gold standard" method in histology (PLM). Consequently, $\mu$ MRI can be used as a quantitative tool to examine tissue properties in individual histological zones, non-destructively. Thirdly, the surfaces of the knee joint seem to be incongruous, with many structural and functional differences between weightbearing and non-weightbearing areas, meniscus covered and meniscus uncovered areas, and peripheral and central areas in tibial cartilage. By analysing the local differences among adjacent samples from the central areas within a single tibia surface, we show that $\mu$ MRI can differentiate topographical variations in tissue degeneration in knee cartilage. Great care should be taken in any study of knee joints because topographical variation in tibia can significantly influence the results in any quantitative study. Fourthly, we show that OA, even in the early stages, can increase the measured values of $T_{2}$ in cartilage. Using the enhanced $T_{2}$ value to monitor the tissue degradation in practice, however, needs a well controlled experimental setting because many instrumental and biological factors can influence the results. An internal $\mathrm{T}_{2}$ reference is probably required practically.

Finally, OA at early stages of the disease is marked with focal lesions of cartilage. We show in this study of an animal model of experimental OA that $\mu \mathrm{MRI}$ is a sensitive imaging technique that can assess ultrastructural changes due to OA. Although achieving the microscopic resolution using the clinical MRI hardware and methodology is currently unlikely, the present results ultimately provide a reachable target for the research community and instrument manufacturers to improve the clinical resolution and to investigate other sensitive sequence/methods to monitor OA progress.

\section{ACKNOWLEDGEMENTS}

YX acknowledges the following funding sources: Research Excellence Fund in Biotechnology from Oakland University, an instrument endorsement from RB and JN Bennett, and an R0l grant (AR 45172) from NIH. JRM is a senior scholar of the Arthritis Society and receives operating support from the Canadian Arthritis Network, the Canadian Institutes of Health Research, and the Natural Science and Engineering Council of Canada.

\section{Authors' affiliations}

H A Alhadlaq, Y Xia, J B Moody, Department of Physics and Center for Biomedical Research, Oakland University, Rochester, MI 48309, USA J R Matyas, Department of Cell Biology and Anatomy, McCaig Center for Joint Injury and Arthritis Research, University of Calgary, Calgary, Alberta T2N 4N1, Canada

\section{REFERENCES}

1 CDC. Press Release. Arthritis and chronic joint symptoms more common than previously thought. Available at: http://www.cdc.gov/od/oc/media/ pressrel/r021024.htm. Accessed 9 March 2004.

2 Mow VC, Setton LA. Mechanical properties of normal and osteoarthritic articular cartilage. In: Brandt KD, Doherty $M$, Lohmander S, eds. Osteoarthritis. Oxford, New York: Oxford University Press, 1998:108-11.

3 Adams ME, Li DK, McConkey JP, Davidson RG, Day B, Duncan CP, et al. Evaluation of cartilage lesions by magnetic resonance imaging at $0.15 \mathrm{~T}$ : comparison with anatomy and concordance with arthroscopy. J Rheumatol 1991; 18:1573-80.

4 Chan WP, Lang P, Stevens MP, Sack K, Majumdar S, Stoller DW, et al. Osteoarthritis of the knee: comparison of radiography, CT, and MR imaging to assess extent and severity. AJR Am J Roentgenol 1991;157:799-806

5 Burstein D, Bashir A, Gray ML. MRI techniques in early stages of cartilage disease. Invest Radiol 2000;35:622-38.

6 Hutton CW, Vennart W. Osteoarthritis and magnetic resonance imaging: potential and problems. Ann Rheum Dis 1995;54:237-43.

7 Burgkart R, Glaser C, Hyhlik-Durr A, Englmeier KH, Reiser M, Eckstein F. Magnetic resonance imaging-based assessment of cartilage loss in severe osteoarthritis: accuracy, precision, and diagnostic value. Arthritis Rheum 2001;44:2072-7.

8 Jones G, Glisson M, Hynes K, Cicuttini F. Sex and site differences in cartilage development: a possible explanation for variations in knee osteoarthritis in later life. Arthritis Rheum 2000;43:2543-9.

9 Xia Y. Relaxation anisotropy in cartilage by NMR microscopy ( $\mu$ MRI) at 14micron resolution. Magn Reson Med 1998;39:941-9.

10 Rubenstein JD, Kim JK, Morova-Protzner I, Stanchev PL, Henkelman RM. Effects of collagen orientation on MR imaging characteristics of bovine articular cartilage. Radiology 1993;188:219-26.

11 Xia Y, Farquhar T, Burton-Wurster N, Lust G. Origin of cartilage laminae in MRI. J Magn Reson Imaging 1997;7:887-94.

12 Xia Y, Moody JB, Burton-Wurster N, Lust G. Quantitative in situ correlation between microscopic MRI and polarized light microscopy studies of articular cartilage. Osteoarthritis Cartilage 2001;9:393-406

13 Pond MJ, Nuki G. Experimentally-induced osteoarthritis in the dog. Ann Rheum Dis 1973;32:387-8.

14 Xia Y, Moody JB, Alhadlaq H, Hu J. Imaging the physical and morphological properties of a multi-zone young articular cartilage at microscopic resolution. $J$ Magn Reson Imaging 2003; 17:365-74.

15 Matyas JR, Adams ME, Huang D, Sandell U. Discoordinate gene expression of aggrecan and type II collagen in experimental osteoarthritis. Arthritis Rheum 1995:38:420-5

16 Mow VC, Proctor CS, Kelly MA. Biomechanics of articular cartilage. In: Frankel VH, ed. Basic biomechanics of the musculoskeletal system. 2nd ed. Philadelphia: Lippincott Williams and Wilkins, 1989:43-4.

17 Alexander RM. Animal mechanics. London: Blackwell Scientific, 1983:67-84

18 Bullough PG, Yawitz PS, Tafra L, Boskey AL. Topographical variations in the morphology and biochemistry of adult canine tibial plateau articular cartilage. J Orthop Res 1985;3:1-16.

19 Motulsky H. Intuitive biostatistics. New York: Oxford University Press, 1995.

20 Xia Y, Moody JB, Alhadlag H, Burton-Wurster N, Lust G. Characteristics of topographical heterogeneity of articular cartilage over the joint surface of a humeral head. Osteoarthritis Cartilage 2002; 10:370-80.

21 Hasty KA, Reife RA, Kang AH, Stuart JM. The role of stromelysin in the cartilage destruction that accompanies inflammatory arthritis. Arthritis Rheum 1990;33:388-97.

22 Matyas JR, Ehlers PF, Huang D, Adams ME. The early molecular natural history of experimental osteoarthritis. I. Progressive discoordinate expression of aggrecan and type II procollagen messenger RNA in the articular cartilage of adult animals. Arthritis Rheum 1999;42:993-1002.

23 Eckstein F, Sittek H, Milz S, Putz R, Reiser M. The morphology of articular cartilage assessed by magnetic resonance imaging (MRI). Reproducibility and anatomical correlation. Surg Radiol Anat 1994; 16:429-38.

24 Ho C, Cervilla V, Kjellin I, Haghigi P, Amiel D, Trudell D, et al. Magnetic resonance imaging in assessing cartilage changes in experimental osteoarthrosis of the knee. Invest Radiol 1992;27:84-90.

25 Watson PJ, Carpenter TA, Hall LD, Tyler JA. Cartilage swelling and loss in a spontaneous model of osteoarthritis visualized by magnetic resonance imaging. Osteoarthritis Cartilage 1996;4:197-207.

26 Appleyard RC, Burkhardt D, Ghosh P, Read R, Cake M, Swain MV, et al. Topographical analysis of the structural, biochemical and dynamic biomechanical properties of cartilage in an ovine model of osteoarthritis. Osteoarthritis Cartilage 2003;11:65-77. 
27 Rubenstein JD, Li JG, Majumdar S, Henkelman RM. Image resolution and signal-to-noise ratio requirements for $M R$ imaging of degenerative cartilage. AJR Am J Roentgenol 1997; 169:1089-96.

28 Clark JM. Variation of collagen fiber alignment in a joint surface: a scanning electron microscope study of the tibial plateau in dog, rabbit, and man. J Orthop Res 1991;9:246-57.

29 Stoop R, Buma P, van der Kraan PM, Hollander AP, Clark Billinghurst R, Robin Poole A, et al. Differences in type II collagen degradation between peripheral and central cartilage of rat stifle joints after cranial cruciate ligament transection. Arthritis Rheum 2000;43:2121-31.
30 Orford CR, Gardner DL, O'Connor P. Ultrastructural changes in dog femoral condylar cartilage following anterior cruciate ligament section. J Anat 1983; 137(Pt 4):653-63

31 Cova M, Toffanin R. MR microscopy of hyaline cartilage: current status. Eur Radiol 2002;12:814-23.

32 Peterfy CG. Imaging of the disease process. Curr Opin Rheumatol 2002;14:590-6.

33 Dardzinski BJ, Mosher TJ, Li S, Van Slyke MA, Smith MB. Spatial variation of $\mathrm{T}_{2}$ in human articular cartilage. Radiology 1997;205:546-50

34 Liess C, Lusse S, Karger N, Heller M, Gluer CC. Detection of changes in cartilage water content using MRI T(2)-mapping in vivo. Osteoarthritis Cartilage 2002;10:907-13.

\section{$\mathrm{ECHO}$}

\section{Cytochrome $\mathbf{P 4 5 0}$ polymorphisms, celecoxib, and warfarin}

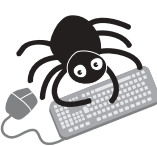

Please visit the Annals of the

Rheumatic

Diseases

website [www. annrheumdis.

com] for a link

to the full text

of this article.
B oth warfarin and non-steroidal anti-inflammatory drugs are metabolised by cytochrome P450 (CYP2C9 enzyme). There are three variants of the CYP2C9 gene $\left(\right.$ CYP $2 C 9^{*} 1,{ }^{*} 2$, and $\left.{ }^{*} 3\right)$ and in European populations about $80 \%$ of people are homozygous for the CYP2C9* 1 allele. The *2 and *3 alleles are found in $11 \%$ and $5-7 \%$ respectively. The * 2 variant is associated with about $12 \%$ of the enzyme activity found with the ${ }^{*} 1$ gene and the ${ }^{*} 3$ variant with $5 \%$ of the activity. Carriers of the variant alleles need smaller doses of warfarin and the concomitant use of drugs that compete for CYP2C9 activity may further reduce warfarin metabolism and result in excessive anticoagulation. Such an occurrence has been described in a case report from New York.

An elderly woman began taking warfarin after having a pacemaker inserted and for a year had an international normalised ratio (INR) for prothrombin time of 2.2-2.6 on a dose of 2.5 mg daily. Within two weeks of taking celecoxib for joint pains the INR had increased to 3.5 . The dose of warfarin was reduced to $1 \mathrm{mg}$ daily but four weeks after starting celecoxib treatment she was admitted to hospital with widespread bleeding into the skin. Her haemoglobin concentration had fallen from 160 to $85 \mathrm{~g} / \mathrm{l}$ over the past week and the INR was $>10$. She was also taking digoxin, ranitidine, and atorvastatin. On analysis of CYP2C9 genotype she was found to be heterozygous for both the ${ }^{*} 2$ and the ${ }^{*} 3$ alleles. She made a complete recovery after stopping both warfarin and celecoxib.

This patient, a heterozygote for both $C Y P 2 C 9^{*} 2$ and $C Y P 2 C 9^{*} 3$ was satisfactorily treated with a modest dose of warfarin but on adding celecoxib to her treatment her anticoagulation became excessive despite a reduction in dose of warfarin. It appears that celecoxib competed with warfarin for P450 enzyme activity which was already genetically low.

A Postgraduate Medical Journal 2004;80:107-109. 